\title{
Microwave-assisted Derivatization of Fatty Acids for Its Measurement in Milk Using High-Performance Liquid Chromatography
}

\author{
Rojeet Shrestha, ${ }^{*}$ Yusuke Miura,* Ken-ichi Hirano, $* *, * * *$ Zhen Chen, ${ }^{*}$ Hiroaki OKabe,* \\ Hitoshi CHIBA, ${ }^{*}$ and Shu-Ping HuI*广 \\ *Faculty of Health Sciences, Hokkaido University, Kita-12, Nishi-5, Sapporo 060-0812, Japan \\ **Laboratory of Cardiovascular Disease, Novel, Non-Invasive, and Nutritional Therapeutics (CNT), \\ Graduate School of Medicine, Osaka University, Osaka 565-0871, Japan \\ ***Department of Cardiovascular Medicine, Graduate School of Medicine, Osaka University, Osaka 565-0871, \\ Japan
}

\begin{abstract}
Fatty acid (FA) profiling of milk has important applications in human health and nutrition. Conventional methods for the saponification and derivatization of FA are time-consuming and laborious. We aimed to develop a simple, rapid, and economical method for the determination of FA in milk. We applied a beneficial approach of microwave-assisted saponification (MAS) of milk fats and microwave-assisted derivatization (MAD) of FA to its hydrazides, integrated with HPLC-based analysis. The optimal conditions for MAS and MAD were determined. Microwave irradiation significantly reduced the sample preparation time from $80 \mathrm{~min}$ in the conventional method to less than $3 \mathrm{~min}$. We used three internal standards for the measurement of short-, medium- and long-chain FA. The proposed method showed satisfactory analytical sensitivity, recovery and reproducibility. There was a significant correlation in the milk FA concentrations between the proposed and conventional methods. Being quick, economic, and convenient, the proposed method for the milk FA measurement can be substitute for the convention method.
\end{abstract}

Keywords Microwave-assisted saponification, microwave-assisted derivatization, fatty acids, milk fats

(Received November 4, 2017; Accepted January 5, 2018; Published May 10, 2018)

\section{Introduction}

Fatty acids (FA) exist in free form or as a component of esters in the living world ubiquitously. The molecular identification and quantification of FA in biological samples have abundant applications in the study of human health and nutrition. The interest of using certain FA as a dietary intervention to benefit various clinical conditions is also increasing. For examples, foods containing polyunsaturated fatty acids (PUFA), such as linoleic acid (FA18:2), linolenic acid (FA18:3), arachidonic acid (FA20:4), eicosapentaenoic acid (EPA, FA20:5) and docosahexaenoic acid (DHA, FA22:6), are generally accepted as protective agents against cardiovascular diseases. ${ }^{1-4}$ Recent studies have revealed the potential clinical utility of mediumchain triglycerides, or the glycerol esters of medium-chain fatty acids (MCFA), in wide varieties of diseases. ${ }^{5-10}$ In most countries, except for those where coconut or palm oils are consumed commonly, only milk and dairy products can provide a significant amount of MCFA. Further, short-chain fatty acids (SCFA) are gathering investigators' interests in microbiome

\footnotetext{
${ }^{\dagger}$ To whom correspondence should be addressed.

E-mail: keino@hs.hokudai.ac.jp

R. S. present address: School of Medicine, Washington University of Barbados, St. Philip, Barbados.
}

studies. Thus, convenient and accurate analytical methods for the quantification of FA, including short-, medium-, and long-chain FA, are desired in the studies of human health and diseases.

Gas chromatography (GC) coupled with detection using flame ionization detector (FID) or mass spectrometer (MS) are currently the most common methods for FA analysis. However, GC is associated with several limitations. Methyl esters of SCFA and MCFA are relatively volatile, and are therefore easily lost in the atmosphere during derivatization and consecutive condensation. This is particularly problematic on analyzing milk samples because of its relatively large contents of SCFA and MCFA. Quantitatively, bovine milk contains 4-12\% of MCFA among the total FA. ${ }^{11,12}$ Secondarily, there is a potent risk for the thermal degradation or structural modification of PUFA during methyl esterification. ${ }^{13}$ Alternatively, highperformance liquid chromatography (HPLC) provides better sensitivity and selectivity in the measurement of FA. ${ }^{13,14}$ HPLC methods using detection by UV/visible, fluorescence, chemiluminescence, electrochemical, light scattering or MS, have been reported for the analysis of FA. ${ }^{4,13,15}$ Because FA are poor chromophores for UV/visible spectrometry, its derivatization is essential. A wide variety of derivatization techniques have been reported for the determination of FA using HPLC. ${ }^{13,16-20}$ Among which, the derivatization of FA to its hydrazide using 2-nitrophenylhydrazine hydrochloride (2-NPH) has been shown to be satisfactory with high sensitivity and 
specificity. ${ }^{16}$ We recently reported a UV-HPLC assay for MCFA in the human serum after labeling the FA with 2-NPH. ${ }^{21}$ We have successfully applied the UV-HPLC method to monitoring the change of free and esterified capric acid (FA10:0) in serum samples after the oral administration of synthetic tricaprin. ${ }^{22}$

The HPLC assay of FA hydrazides has enabled simple, sensitive, and specific measurements of FA. However, derivatization itself is a time-consuming process that involves almost $1 \mathrm{~h}$ of heating in a water bath (classical heating method). ${ }^{21}$ Microwave-assisted heating exhibits the advantages to accelerate the rate of chemical reactions through thermal effect due to microwave absorption by polar substances. ${ }^{23-28}$ In the past few decades, the use of microwaves in the organic synthesis and derivatization for GC/LC analysis is gradually increasing, replacing the conventional heating techniques. The major advantage of microwave irradiation is that it generates efficient internal heating by the direct coupling of electromagnetic waves with the molecules, particularly dipole or ionic molecules, present in the reaction mixture. ${ }^{29}$ This results in an instantaneous transfer of energy and a rapid rise of the temperature and thus provides reaction homogeneity, which otherwise cannot be achieved by the classical heating methods. As a consequence, it accelerates the rate of chemical reactions, and significantly reduces the reaction completion time. Besides this, non-thermal effects of microwave radiation, like molecular mobility and field stabilization, may also contribute to enhance the chemical reactions. $^{24}$ Furthermore, MAD has been demonstrated to improve the yields and formation of cleaner products, thereby decreasing the effects of interfering substances. ${ }^{30}$ Microwave irradiation has been reported to be useful in wide varieties of laboratory techniques. ${ }^{31,32}$ Microwave heating has been successfully used in wide varieties of derivatization techniques for quantitative analysis..$^{30,33-35}$ MAD has become a part of routine operating procedures in many laboratories, and been used in the GC/MS analysis of steroids, ${ }^{34,36}$ phenolic acids, $, 37,38$ fatty acids,,$^{39,40}$ and urinary metabolites..$^{30,41,42}$ Moreover, microwave irradiation can also enhance the hydrolysis of the esters bond during the saponification of milk samples. Therefore, we aimed to apply microwave irradiation for the derivatization of FA for HPLC. In this report, we describe an optimal condition for microwave-assisted saponification (MAS) and microwave-assisted derivatization (MAD) of FA with 2-NPH. We show the analytical performance of the measurement of milk FA by using a microwave-assisted sample pretreatment.

\section{Experimental}

\section{Reagents and chemicals}

2-Nitrophenylhydrazine hydrochloride (2-NPH$\cdot \mathrm{HCl})$, 1-(3-dimethylaminopropyl)-3-ethylcarbodiimide hydrochloride (1-EDC.HCl), butyric acid (FA4:0), hexanoic acid (FA6:0), heptanoic acid (FA7:0), octanoic acid (FA8:0), undecanoic acid (FA11:0), lauric acid (FA12:0), myristic acid (FA14:0), palmitic acid (FA16:0), heptadecanoic acid (margaric acid, FA17:0), stearic acid (FA18:0), nonadecanoic acid (FA19:0), arachidic acid (FA20:0), tricosanoic acid (FA23:0), linoleic acid (FA18:2), and linolenic acid (FA18:3) were purchased from Tokyo Chemical Industry Co., Ltd (Tokyo, Japan). Oleic acid (FA18:1), arachidonic acid (FA20:4), eicosapentaenoic acid (EPA, FA20:5) and docosahexaenoic acid (DHA, FA22:6) were obtained from Sigma-Aldrich Japan (Tokyo, Japan). Capric acid (FA10:0), and HPLC grade methanol and water were from Wako Pure Chemical Industry (Osaka, Japan). FA labeling reagents were obtained from YMC CO (Kyoto, Japan).

\section{Synthesis of standards}

We used 2-NPH-labeled FA4:0, FA6:0, FA8:0, FA10:0, FA12:0, FA14:0, FA16:0, FA18:0, FA19:0, FA20:0, FA22:0, FA23:0, FA18:1, FA18:2, FA18:3, FA20:4, FA20:5 and FA22:6 as standards to identify and determine the retention time (RT) of FA in HPLC. Each standard was synthesized in our laboratory by the method as previously reported..$^{21}$ 1,2,3-Triundecanoylglycerol (TG11:0/11:0/11:0) and 1,2,3-trinonadecanoylglycerol (TG19:0/19:0/19:0) used as internal standards (IS) were synthesized in our laboratory. The purity of each standard and IS were assessed by ${ }^{1} \mathrm{H}$ and ${ }^{13} \mathrm{C}$-nuclear magnetic resonance (NMR) using JEOL JNM-AL400 (Tokyo, Japan) with $\mathrm{CDCl}_{3}$ as a solvent (data not shown). The molecular characterizations of synthesized FA-NPH and IS were carried out by an electrospray ionization ion-trap mass spectrometer (Thermo Scientific Finnigan LXQ, CA, USA).

\section{Preparation of standard curve}

Working standards of each NPH labeled FA were prepared to give final concentrations of $0.1,1.0,2.0,4.0,6.0,8.0$, $20.0 \mathrm{pmol} / \mu \mathrm{L}$. FA7:0 was used as IS for the calibration of FA4:0, FA6:0, and FA8:0. Triundecanoylglycerol was used as IS for the determination of FA10:0, FA12:0 and FA14:0, while trinonadecanoylglycerol was used for the calibration of remaining longer chain FA. The standard solutions were stored at $-20^{\circ} \mathrm{C}$ and allowed to equilibrate at room temperature before use. Calibration curves were constructed by plotting the peak area ratio of each FA-NPH standard to its corresponding IS against its concentration.

\section{Milk samples}

We applied the proposed method to evaluate FA profiling in bovine milk available from commercial companies over different regions in Japan. All milk samples were stored at $-80^{\circ} \mathrm{C}$ and homogenized thoroughly by mixing before use. To compare the analytical performance of MAD with conventional heating, a total of 33 milk samples were analyzed.

\section{Optimization of microwave-assisted saponification}

For the optimization of saponification using microwave heating, $3 \mu \mathrm{L}$ of each milk sample was spiked with TG19:0/19:0/19:0 at 3 different quantities, making the final concentration of FA19:0 to be 1000, 10000 and $20000 \mathrm{~mol} / \mathrm{L}$. FA23:0 (10 nmol) was used as IS in this step. After treating the spiked milk sample with $100 \mu \mathrm{L} \mathrm{KOH}(0.3 \mathrm{~N}$ in ethanol), the mixture was exposed to microwaves for different time durations, ranging from 10 to $300 \mathrm{~s}$. In the conventional method, saponification was carried out by heating the mixture at $80^{\circ} \mathrm{C}$ for $45 \mathrm{~min}$. The saponified products were derivatized using the conventional method and analyzed by HPLC, as previously reported. ${ }^{21}$ The analysis was performed 5 times for each timeduration. The concentrations of FA19:0 in the spiked samples saponified by microwave and conventional heating were compared.

\section{Optimization of microwave-assisted derivatization}

To evaluate MAD, a mixture of standards including IS, each concentration of 2.0 and $5.0 \mathrm{nmol}$, was treated with labeling reagents and exposed to microwaves for $5,10,20,30,40,60$, and $90 \mathrm{~s}$. The same concentration of the standard was also processed with the conventional heating method. The experiment was repeated 5 times and mean peak area of each FA hydrazides generated from MAD was compared to that from conventional heating. 


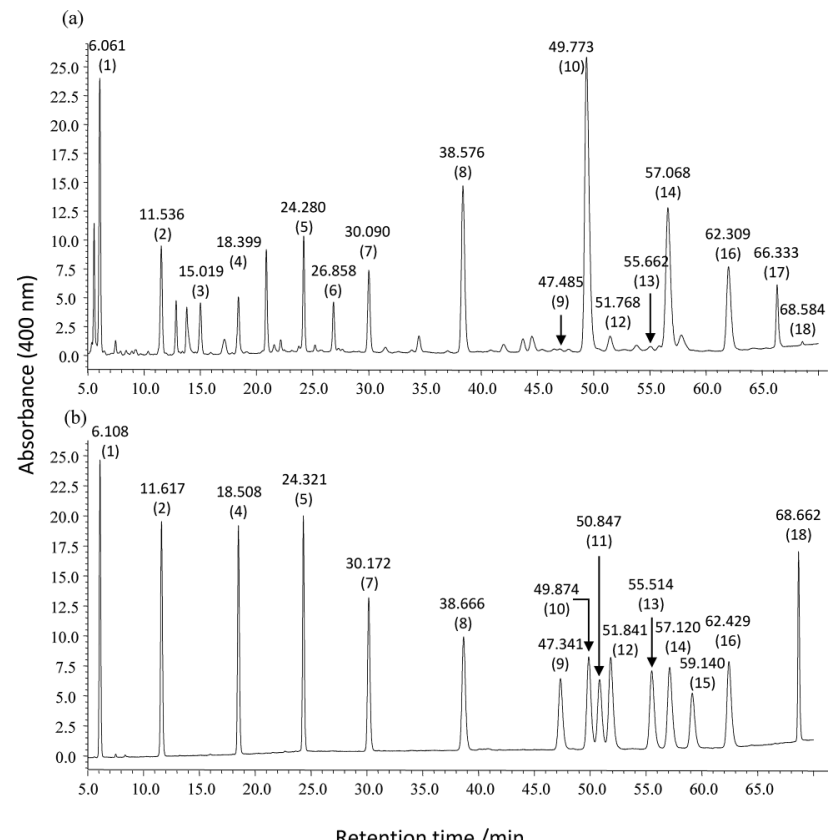

Fig. 1 HPLC chromatogram of 2-nitrophenylhydrazine derivatives of (a) fatty acids in a milk sample obtained by microwave-assisted derivatization and (b) mixture of standard fatty acids. Peak: 1, butyric acid (FA4:0); 2, hexanoic acid (FA6:0); 3, heptanoic acid (FA7:0, Internal standard-1); 4, octanoic acid (FA8:0); 5, capric acid (FA10:0); 6, undecanoic acid (FA11:0, Internal standard-2); 7, lauric acid (FA12:0); 8, myristic acid (FA14:0); 9, linolenic acid (FA18:3); 10, palmitic acid (FA16:0); 11, eicosapentaenoic acid (FA20:5, EPA); 12 , linoleic acid (FA18:2); 13, arachidonic acid (FA20:4); 14, oleic acid (FA18:1); 15, docosahexaenoic acid (FA22:6, DHA), 16, stearic acid (FA18:0); 17, nonadecanoic acid (FA19:0, Internal standard-3); and 18, arachidic (FA20:0).

\section{Assay of FA}

In this study, we replaced the conventional heating in a water bath for the saponification and derivatization of FA with microwave irradiation. To determine the total fatty acids (esterified plus non-esterified), the MAS was carried out. For this, $3.0 \mu \mathrm{L}$ of milk sample was mixed with FA7:0 $(2.0 \mathrm{nmol})$, TG11:0/11:0/11:0 (3.0 nmol), and TG19:0/19:0/19:0 (4.0 nmol) as IS in a screw-cap glass tubes. Into the mixture, $100 \mu \mathrm{L}$ of $0.3 \mathrm{~N} \mathrm{KOH}$ was added and was exposed to microwave irradiation in a domestic $1200 \mathrm{~W}(50 \mathrm{~Hz})$ microwave oven (Neove, Tokyo, Japan) for $150 \mathrm{~s}$ to ensure complete saponification of esterified FA present in the sample.

For the determination of free FA (non-esterified FA), $100 \mu \mathrm{L}$ of milk sample was mixed with IS FA7:0 (2.0 nmol), IS FA11:0 $(2.0 \mathrm{nmol})$ and IS FA19:0 (4.0 nmol), and processed further without any saponification. The remaining procedures were the same between the assays for free and total FA. The MAD was achieved by the addition of $200 \mu \mathrm{L}$ of each 2-NPH. $\mathrm{HCl}(20 \mathrm{mM}$ in ethanol) and 1-EDC. $\mathrm{HCl}(0.25 \mathrm{M}$ in ethanol:pyridine, 97:3 $(\mathrm{v} / \mathrm{v}))$, followed by the microwave irradiation for $10 \mathrm{~s}$. In the conventional method, the labeling was performed by incubating the mixture at $60^{\circ} \mathrm{C}$ for $20 \mathrm{~min}$. The extraction of derivatized FA was done using $4 \mathrm{~mL}$ each of diethyl ether and potassium phosphate buffer ( $\mathrm{pH}$ 4.6). The mixture was then vortex-mixed vigorously for $30 \mathrm{~s}$ and centrifuged at $3000 \times g$ for $30 \mathrm{~min}$ at $25^{\circ} \mathrm{C}$. The upper layer of diethyl ether extract was collected using a glass pipette, and completely evaporated in a centrifugal concentrator (Tomy centrifugal concentrator, Tokyo, Japan). The residue was dissolved in $200 \mu \mathrm{L}$ of methanol and filtered using a centrifugal filtration device (PVDF $0.1 \mu \mathrm{m}$, Merck Millipore Ltd., Carrigtwohill, Ireland). Ten microliters of the extract were injected into HPLC column.

\section{Liquid chromatography conditions}

HPLC analyses were performed in a Shimadzu Nexera X2 LC-30AD UHPLC (Shimadzu Seisakusho, Kyoto, Japan) equipped with a Shimadzu Nexera X2 SIL-30AC autosampler, CTO-20A Prominence column oven and SPD-M20A photo diode array detector. The absorbance was measured at $400 \mathrm{~nm}$. A series of experiments were performed to select the optimal HPLC conditions in order to identify the best column, optimal solvents and its elution profiles for the separation of all FA along with IS. We found that Kinetex Biphenyl reversed phase column $(250 \times 4.6 \mathrm{~mm}$, i.d.; particle size $5 \mu \mathrm{m}$, Phenomenex, CA, USA), maintained at $35^{\circ} \mathrm{C}$ is the best column for a highly efficient separation of FA with carbon number $\mathrm{C} 4$ to $\mathrm{C} 20$. Gradient elution was performed with water adjusted to $\mathrm{pH} 4.0$ with $1 \%(\mathrm{v} / \mathrm{v})$ trifluoroacetic acid (solvent $\mathrm{A})$ and methanol (solvent B), at a flow rate of $1.0 \mathrm{~mL} / \mathrm{min}$. The HPLC gradient was programmed as follows: $0.00-0.20 \mathrm{~min}$ isocratic at $50 \%$ B, $0.20-30.00 \min 50 \rightarrow 78 \%$ B, $30.00-50.00 \min 78 \rightarrow 82 \%$ B, $50.00-60.00 \min 82 \rightarrow 100 \%$ B. Five-minutes of washing with $100 \%$ methanol were necessary after each run to wash out any residual peaks. Typical chromatograms obtained from a milk sample and the standards are shown in Figs. 1(a) and 1(b), respectively.

\section{Evaluation of extraction solvent}

We compared the extraction recoveries for FA hydrazides among chloroform, hexane, and diethyl ether. Chloroform showed relatively higher recoveries for the hydrazides of all saturated FA, while the recoveries of hydrazides of PUFA was not satisfactory. Furthermore, the chloroform extract showed a significant number of interfering peaks in the chromatogram of milk samples. On the other hand, the hexane extracts showed good recoveries for all LCFA including PUFA, and was free from interference. However, it showed poor recoveries for SCFA. We selected diethyl ether as the best extraction solvent, since it showed an acceptable performance in the extraction of all FA hydrazides.

\section{Accuracy, precision, and recovery}

The recovery and reproducibility for the FA assay were studied by repeating the analysis of known concentrations of all FA for 6 times in a run (intra-assay) and 6 different runs (interassay). The inter- and intra-assay coefficients of variation (CV) were determined at low, average and high concentrations selected on the basis of the milk FA concentration.

\section{Results and Discussion}

\section{Evaluation of microwave-assisted saponification}

In order to ensure complete saponification of lipids using microwave heating, milk samples spiked with TG19:0/19:0/19:0 at 3 different concentrations were analyzed 5 times under different reaction times, ranging from 10 to $300 \mathrm{~s}$. The results of evaluating the saponification are shown in Fig. 2. The spiked FA19:0 concentration was recovered within $150 \mathrm{~s}$ of microwave irradiation, suggesting that complete saponification was achieved after this period. Furthermore, the concentration of all fatty acids obtained after saponification for $150 \mathrm{~s}$ under microwave was comparable to that of conventional heating at $80^{\circ} \mathrm{C}$ for 45 min (Fig. 2). 

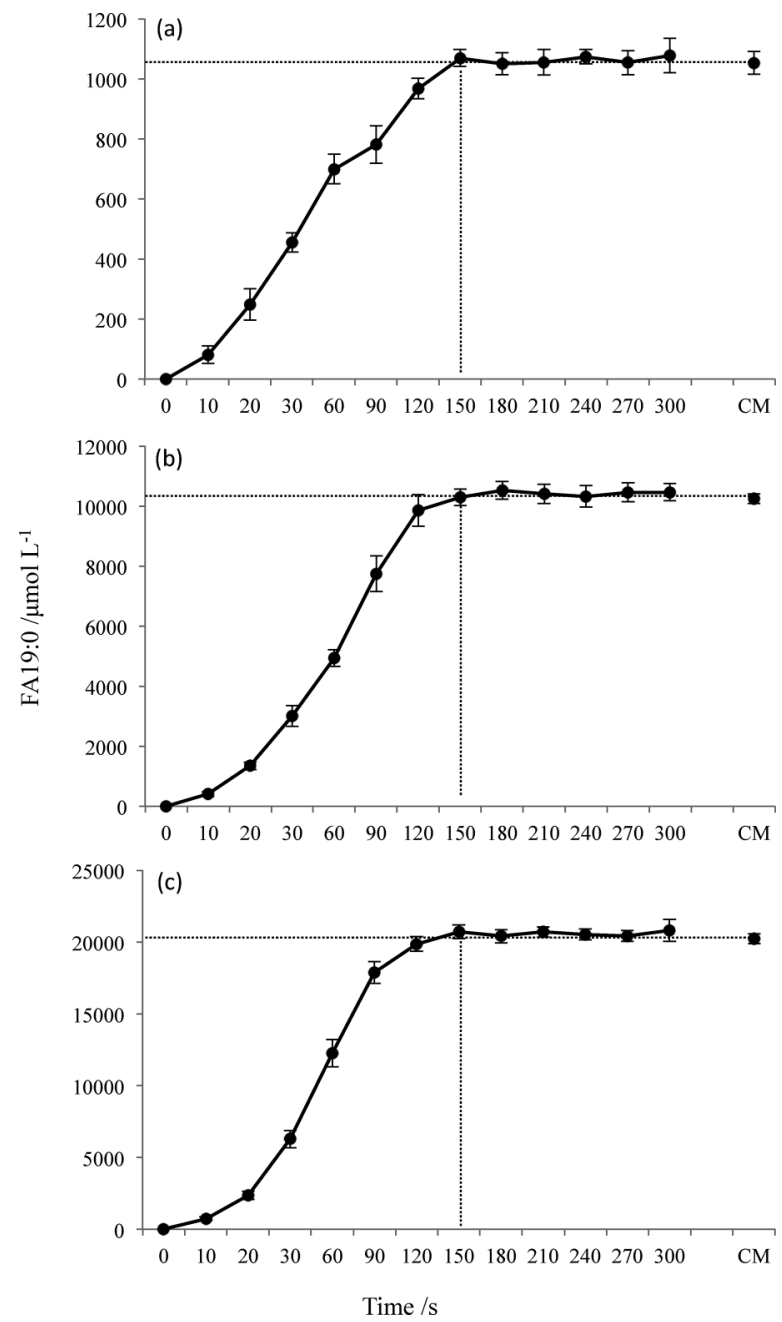

Fig. 2 Effect of the reaction time under microwave-assisted heating on the saponification of spiked 1,2,3-trinonadecanoylglycerol (TG19:0/19:0/19:0) in a milk sample $(n=5)$ to release free nonadecanoic acid (FA19:0) (a) final concentration of $1000 \mu \mathrm{mol} / \mathrm{L}$; (b) final concentration of $10000 \mu \mathrm{mol} / \mathrm{L}$; (c) final concentration of $20000 \mu \mathrm{mol} / \mathrm{L}$. CM: conventional heating method.

\section{Evaluation of microwave-assisted derivatization}

The derivatization of FA with NPH using microwave heating was evaluated by monitoring the peak area generated after reacting 9 species of FA including 3 IS at two different concentrations of 2.0 and $5.0 \mathrm{nmol}$ under various reactions time $(n=5)$. Figure 3 shows the relationship between the average peak area of fatty acid hydrazides and the reaction time (5 to $90 \mathrm{~s}$ ) under microwave irradiation. The peak area increased and attended maximum with $10 \mathrm{~s}$ of microwave exposure. A prolonged reaction beyond $30 \mathrm{~s}$ is not recommended, since it is associated with a decrease in the signal of acid hydrazides. Microwave-assisted heating for $10 \mathrm{~s}$ is adequate for the maximum derivatization of MCFA (Fig. 3(a)), LCFA (Fig. 3(b)), and internal standards (Fig. 3(c)) and generates comparable amounts of acid hydrazides as generated by conventional heating at $60^{\circ} \mathrm{C}$ for $20 \mathrm{~min}$. Therefore, our result suggests that $10 \mathrm{~s}$ of microwave irradiation generates a similar peak area to that of conventional heating without compromising the reproducibility.

Calibration, recovery and reproducibility

An eight-point calibration curve ranging from 1 to $200 \mathrm{pmol}$

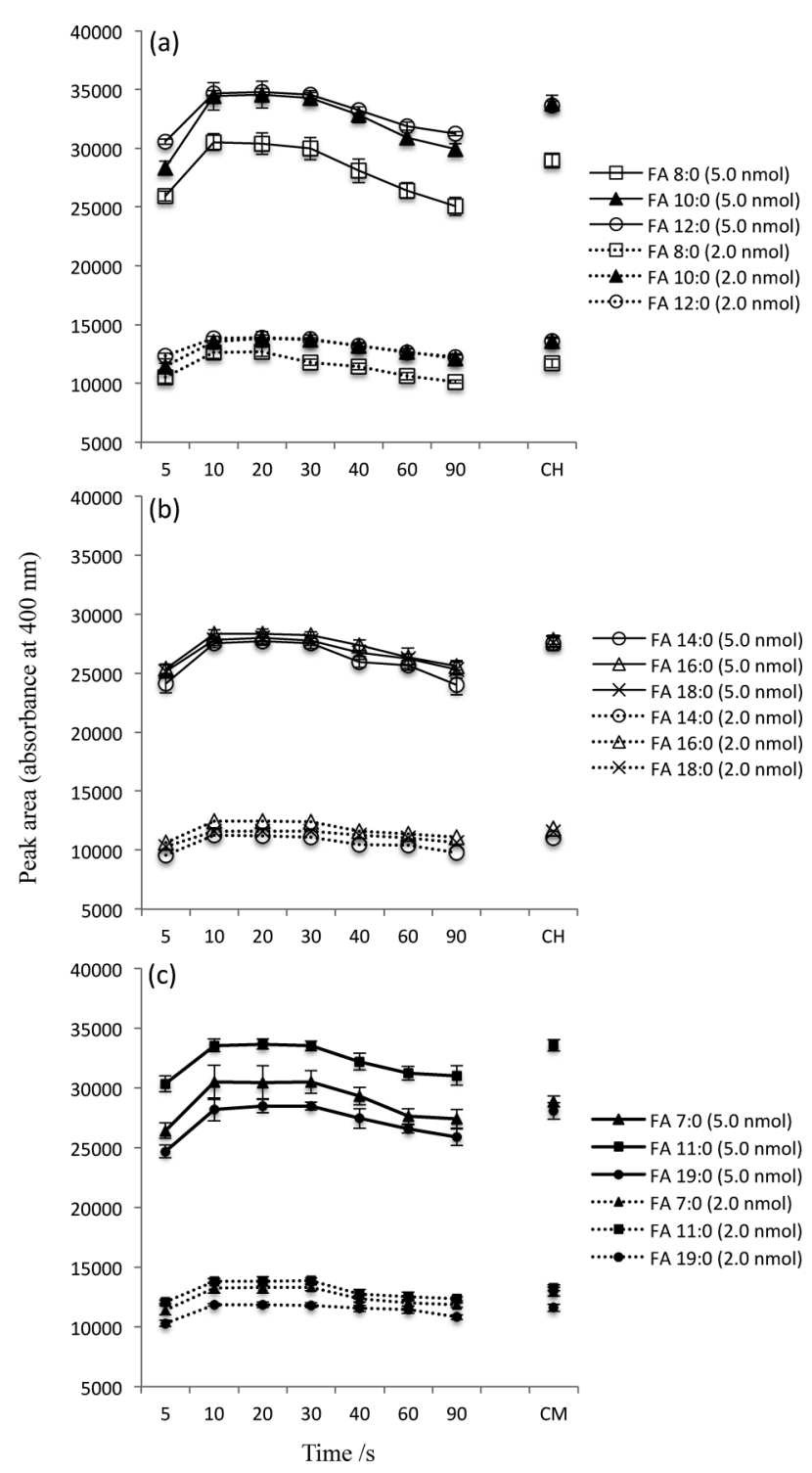

Fig. 3 Effect of the reaction time under microwave-assisted heating on the formation of NPH labeled fatty acids at two different concentrations $(n=5)$. (a) Medium-chain fatty acids; (b) long-chain fatty acids; (c) internal standards. CM: conventional heating method.

per injection for NPH derivatives of each FA was evaluated. When $10 \mu \mathrm{L}$ of the above-prepared calibration specimen was injected $(0.1,1.0,2.0,4.0,6.0,8.0$, and $20.0 \mathrm{pmol} / \mu \mathrm{L})$ on the column, the response $(y)$ was linearly related to FA concentration (x) (Supporting Information; Fig. S1). Each calibration curve, as determined by linear regression analysis, showed good linearity with the correlation coefficient $\left(r^{2}\right)$ above 0.99 for all FA. The limits of detection (LOD) were 1 pmol for all FA at signal-to-noise ratio $(S / N)$ of $4: 1$. The lower limit of quantitation (LOQ) was 3 pmol for each FA at $S / N$ 5:1 per injection to the column.

The data of the inter-assay and intra-assay variations for the FA analysis are given in Table $1 . \mathrm{CV}$ of imprecision were within 0.3 to $10 \%$ for all FA in both inter- and intra-assay run. PUFA, particularly at low concentration show a relatively higher degree of imprecision. The recoveries of all FA were within 85.1 to $108.5 \%$ in inter-assay and 87.1 to $109.3 \%$ in intra-assay runs (Table 1). Based on these data, our method is suitable for the measurement of FA in milk. 
Table 1 Inter- and intra-assay precision and accuracy of fatty acids quantification and recovery of fatty acids spiked in the samples

\begin{tabular}{|c|c|c|c|c|c|c|c|c|c|}
\hline \multirow[b]{2}{*}{ Fatty acid } & \multirow[b]{2}{*}{$\begin{array}{c}\text { Expected } \\
\text { conc./ } \\
\mu \mathrm{mol} \mathrm{L}{ }^{-1}\end{array}$} & \multicolumn{4}{|c|}{ Inter-assay $(n=6)$} & \multicolumn{4}{|c|}{ Intra-assay $(n=6)$} \\
\hline & & $\begin{array}{c}\text { Observed } \\
\text { concentration } \\
\text { Mean } \pm \mathrm{SD} / \\
\mu \mathrm{mol} \mathrm{L}-1\end{array}$ & $\begin{array}{c}\text { Precision } \\
\text { [CV], } \\
\%\end{array}$ & $\begin{array}{l}\text { Recovery } \\
(\% \text { of } \\
\text { expected } \\
\text { value })\end{array}$ & $\begin{array}{c}\text { Recovery } \\
\text { range, } \\
\%\end{array}$ & $\begin{array}{c}\text { Observed } \\
\text { concentration } \\
\text { Mean } \pm \mathrm{SD} / \\
\mu \mathrm{mol} \mathrm{L}-1\end{array}$ & $\begin{array}{c}\text { Precision } \\
{[\mathrm{CV}]} \\
\%\end{array}$ & $\begin{array}{l}\text { Recovery } \\
(\% \text { of } \\
\text { expected } \\
\text { value })\end{array}$ & $\begin{array}{c}\text { Recovery } \\
\text { range, } \\
\%\end{array}$ \\
\hline \multirow{3}{*}{$\begin{array}{l}\text { Butyric acid } \\
\text { (FA 4:0) }\end{array}$} & 500.0 & $537 \pm 21.4$ & 4.0 & 107.5 & $102.8-113.5$ & $521.2 \pm 30.7$ & 5.9 & 104.2 & $98.4-107.2$ \\
\hline & 1000.0 & $1033.6 \pm 51.0$ & 4.9 & 103.4 & $94.6-107.1$ & $1024.1 \pm 77.4$ & 7.6 & 102.4 & $92.3-113.4$ \\
\hline & 2000.0 & $2051.3 \pm 26.8$ & 1.3 & 102.6 & $100.6-103.9$ & $2027.9 \pm 76.1$ & 3.8 & 101.4 & $95.8-106.6$ \\
\hline \multirow{3}{*}{$\begin{array}{l}\text { Hexanoic acid } \\
\text { (FA 6:0) }\end{array}$} & 250.0 & $259.3 \pm 8.8$ & 3.4 & 103.7 & $98.7-108.0$ & $256.1 \pm 18.4$ & 7.2 & 102.5 & $94.3-113.1$ \\
\hline & 500.0 & $506.5 \pm 24.0$ & 4.7 & 101.3 & $96.4-109.7$ & $518.0 \pm 40.4$ & 7.8 & 103.6 & $94.2-113.8$ \\
\hline & 1000.0 & $1037.7 \pm 39.8$ & 3.8 & 103.8 & $97.3-108.5$ & $1033.9 \pm 71.5$ & 6.9 & 103.4 & $95.4-112.6$ \\
\hline \multirow{3}{*}{$\begin{array}{l}\text { Octanoic acid } \\
\text { (FA 8:0) }\end{array}$} & 250.0 & $248.5 \pm 8.3$ & 3.3 & 99.4 & $95.8-104.1$ & $250.9 \pm 12.1$ & 4.8 & 100.4 & $95.6-107.5$ \\
\hline & 500.0 & $505.5 \pm 9.8$ & 1.9 & 101.1 & $98.9-104.5$ & $509.0 \pm 20.6$ & 4.0 & 101.8 & $97.4-106.7$ \\
\hline & 1000.0 & $1041.0 \pm 22.8$ & 2.2 & 104.1 & $100.9-106.1$ & $1034.8 \pm 38.2$ & 3.7 & 103.5 & $98.6-108.7$ \\
\hline \multirow{3}{*}{$\begin{array}{l}\text { Capric acid } \\
\text { (FA10:0) }\end{array}$} & 500.0 & $499.7 \pm 45.9$ & 9.2 & 99.9 & $83.8-108.6$ & $523 \pm 40.6$ & 7.8 & 104.6 & $92.2-112.3$ \\
\hline & 1000.0 & $990.0 \pm 58.7$ & 5.9 & 99.0 & $91.3-107.6$ & $1029 \pm 87.2$ & 8.5 & 102.0 & $92.6-112.5$ \\
\hline & 2000.0 & $1983.6 \pm 128.3$ & 6.5 & 99.2 & $96.5-110.8$ & $2095.6 \pm 141.9$ & 6.8 & 104.8 & $92.7-112.2$ \\
\hline \multirow{3}{*}{$\begin{array}{l}\text { Lauric acid } \\
\text { (FA 12:0) }\end{array}$} & 500.0 & $544.8 \pm 30.0$ & 5.5 & 109.0 & $97.3-114.0$ & $525.2 \pm 47.8$ & 9.1 & 105 & $93.4-114.3$ \\
\hline & 1000.0 & $1042.7 \pm 65.5$ & 6.3 & 104.3 & $95.4-114.3$ & $997.6 \pm 88.5$ & 8.9 & 99.8 & $85.3-110.4$ \\
\hline & 2000.0 & $2050.6 \pm 108.2$ & 5.3 & 102.5 & $96.8-112.1$ & $2186.2 \pm 146.2$ & 6.7 & 109.3 & $99.2-115.6$ \\
\hline \multirow{3}{*}{$\begin{array}{l}\text { Myristic acid } \\
\text { (FA 14:0) }\end{array}$} & 1000.0 & $1078.5 \pm 74.8$ & 6.9 & 107.8 & $96.9-115.6$ & $1074.3 \pm 81.8$ & 7.6 & 107.4 & $95.5-116.7$ \\
\hline & 2000.0 & $2089.6 \pm 139.9$ & 6.7 & 104.5 & $97.6-115.5$ & $2016.2 \pm 165.3$ & 8.2 & 100.8 & $89.8-112.4$ \\
\hline & 4000.0 & $4034.7 \pm 219.4$ & 5.4 & 100.9 & $94.2-108.9$ & $4257.2 \pm 228.7$ & 5.4 & 106.4 & $100.6-114.6$ \\
\hline \multirow{3}{*}{$\begin{array}{l}\text { Palmitic acid } \\
\text { (FA16:0) }\end{array}$} & 2000.0 & $2011.3 \pm 7.0$ & 0.3 & 100.6 & $99.9-100.9$ & $2021.6 \pm 59.6$ & 2.9 & 101.1 & $97.7-103.9$ \\
\hline & 4000.0 & $3977.1 \pm 45.0$ & 1.1 & 99.4 & $97.5-100.8$ & $3989.0 \pm 86.0$ & 2.2 & 99.7 & $97.1-102.2$ \\
\hline & 6000.0 & $5971.2 \pm 41.6$ & 0.7 & 99.5 & $98.8-100.9$ & $5995.1 \pm 146.8$ & 2.4 & 99.9 & $96.4-103.6$ \\
\hline \multirow{3}{*}{$\begin{array}{l}\text { Stearic acid } \\
\text { (FA18:0) }\end{array}$} & 1000.0 & $1027.5 \pm 16.9$ & 1.6 & 102.7 & $100.7-104.5$ & $1023.6 \pm 38.8$ & 3.8 & 102.4 & $97.5-107.9$ \\
\hline & 2000.0 & $1978.7 \pm 22.0$ & 1.1 & 98.9 & $97.5-100.8$ & $1957.5 \pm 37.2$ & 1.9 & 97.9 & $95.4-100.3$ \\
\hline & 4000.0 & $3858.2 \pm 44.8$ & 1.2 & 96.5 & $95.5-98.4$ & $3835.8 \pm 117.6$ & 3.1 & 95.9 & $91.4-99.2$ \\
\hline \multirow{3}{*}{$\begin{array}{l}\text { Oleic acid } \\
\text { (FA 18:1) }\end{array}$} & 1000.0 & $1009.1 \pm 5.9$ & 0.6 & 100.9 & $100.1-101.9$ & $999.4 \pm 25.8$ & 2.6 & 99.9 & $96.4-103.5$ \\
\hline & 2000.0 & $2035.1 \pm 51.0$ & 2.5 & 101.8 & $97.9-105.3$ & $2009.3 \pm 58.4$ & 2.9 & 100.5 & $97.1-104.8$ \\
\hline & 4000.0 & $4110.5 \pm 56.4$ & 1.4 & 102.8 & $100.6-104.1$ & $4107.1 \pm 113.0$ & 2.8 & 102.7 & $99.5-105.7$ \\
\hline \multirow{3}{*}{$\begin{array}{l}\text { Linoleic acid } \\
\text { (FA 18:2) }\end{array}$} & 100.0 & $101.7 \pm 10.8$ & 10.6 & 101.7 & $81.8-116.7$ & $96.3 \pm 9.8$ & 10.2 & 96.3 & $84.2-110.6$ \\
\hline & 500.0 & $482.2 \pm 8.9$ & 1.8 & 96.4 & $93.7-98.3$ & $457.0 \pm 23.4$ & 5.1 & 91.4 & $86.4-99.5$ \\
\hline & 1000.0 & $999.4 \pm 21.9$ & 2.2 & 99.9 & $97.7-102.9$ & $973.6 \pm 50.7$ & 5.2 & 97.4 & $89.9-105.6$ \\
\hline \multirow{3}{*}{$\begin{array}{l}\text { Linolenic acid } \\
\text { (FA 18:3) }\end{array}$} & 100.0 & $98.1 \pm 11.9$ & 12.1 & 98.1 & $84.9-116.7$ & $96.6 \pm 10.0$ & 10.3 & 96.6 & $83.9-111.6$ \\
\hline & 500.0 & $425.4 \pm 29.3$ & 6.9 & 85.1 & $75.0-92.1$ & $435.6 \pm 38.4$ & 8.8 & 87.1 & $78.2-98.4$ \\
\hline & 1000.0 & $980.1 \pm 57.5$ & 5.9 & 98.0 & 92.9- 106.9 & $971.0 \pm 63.3$ & 6.5 & 97.1 & $89.7-106.5$ \\
\hline \multirow{3}{*}{$\begin{array}{l}\text { Arachidonic acid } \\
\text { (FA 20:4) }\end{array}$} & 100.0 & $104.2 \pm 9.0$ & 8.6 & 104.2 & $95.7-120.4$ & $105.4 \pm 9.7$ & 9.2 & 105.4 & $90.6-115.8$ \\
\hline & 500.0 & $507.1 \pm 21.6$ & 4.3 & 101.4 & $93.7-106.6$ & $491.7 \pm 34.0$ & 6.9 & 98.3 & $89.9-109.1$ \\
\hline & 1000.0 & $1084.6 \pm 31.2$ & 2.9 & 108.5 & $103.3-112.4$ & $1066.6 \pm 45.1$ & 4.2 & 106.7 & $98.8-110.9$ \\
\hline EPA & 100.0 & $94.9 \pm 10.9$ & 11.5 & 94.9 & $80.0-113.0$ & $97.6 \pm 8.9$ & 9.1 & 97.6 & $88.1-114.2$ \\
\hline \multirow[t]{2}{*}{ (FA 20:5) } & 500.0 & $476.2 \pm 19.5$ & 4.1 & 95.2 & $89.9-100.3$ & $465.7 \pm 22.7$ & 4.9 & 93.1 & $87.0-100.4$ \\
\hline & 1000.0 & $1017.8 \pm 39.1$ & 3.9 & 101.8 & $95.4-105.3$ & $977.8 \pm 46.0$ & 4.7 & 97.8 & $92.4-103.2$ \\
\hline DHA & 100.0 & $92 \pm 5.0$ & 5.4 & 92.0 & $83.8-98.1$ & $92.3 \pm 6.7$ & 7.2 & 92.3 & $80.6-99.1$ \\
\hline \multirow[t]{2}{*}{ (FA 22:6) } & 500.0 & $463.2 \pm 28.7$ & 6.2 & 92.6 & $85.3-98.8$ & $464.7 \pm 30.4$ & 6.5 & 92.9 & $84.5-100.3$ \\
\hline & 1000.0 & $1017.8 \pm 46.2$ & 4.5 & 101.8 & $93.2-107.0$ & $1001.1 \pm 64.4$ & 6.4 & 100.1 & $92.3-110.3$ \\
\hline
\end{tabular}

Comparison with conventional method

We measured the FA concentration in 33 milk samples after MAS and MAD, and compared the results with that from the conventional thermal incubation method. A statistical comparison of the results by our microwave-assisted method with the conventional method indicates that these methods correlated well with a Spearman rank correlation coefficient $(r)$ above 0.97 for all FA $(p<0.001)$ (Fig. 4). Therefore, there was a significant association between the proposed and conventional methods.

This study provides the first approach to use MAS of milk and MAD of FA to corresponding FA hydrazides coupled with its HPLC analysis. In this study, MAD was used to accelerate the chemical reaction rather than by the conventional heating. Because of the dipole movement of polar groups of reactants and aqueous alcohol solvent system, MAD can be easily applied in the derivatization, since they strongly absorb microwave energy. We found that MAD of FA with 2-NPH have remarkable performance and can be applied to FA profiling in the milk samples.

There are several noteworthy merits of this analytical approach. First, the overall time for the preparation of samples has been significantly reduced in the present method. In our previously published report, ${ }^{22}$ the pretreatment of samples using the conventional heating method takes $80 \mathrm{~min}$, which can be shortened to $160 \mathrm{~s}$ using microwave heating. The derivatization alone needs $20 \mathrm{~min}$ of heating at $60^{\circ} \mathrm{C}$ in the conventional method, which can be decreased to $10 \mathrm{~s}$ using MAD. Certainly, this is a huge reduction in the sample preparation time. Moreover, in the conventional method, it was necessary to further incubate the derivatized sample with $\mathrm{KOH}(10 \%$, w/v in methanol: water $=1: 1$ ) at $60^{\circ} \mathrm{C}$ for $15 \mathrm{~min}$ to remove the 


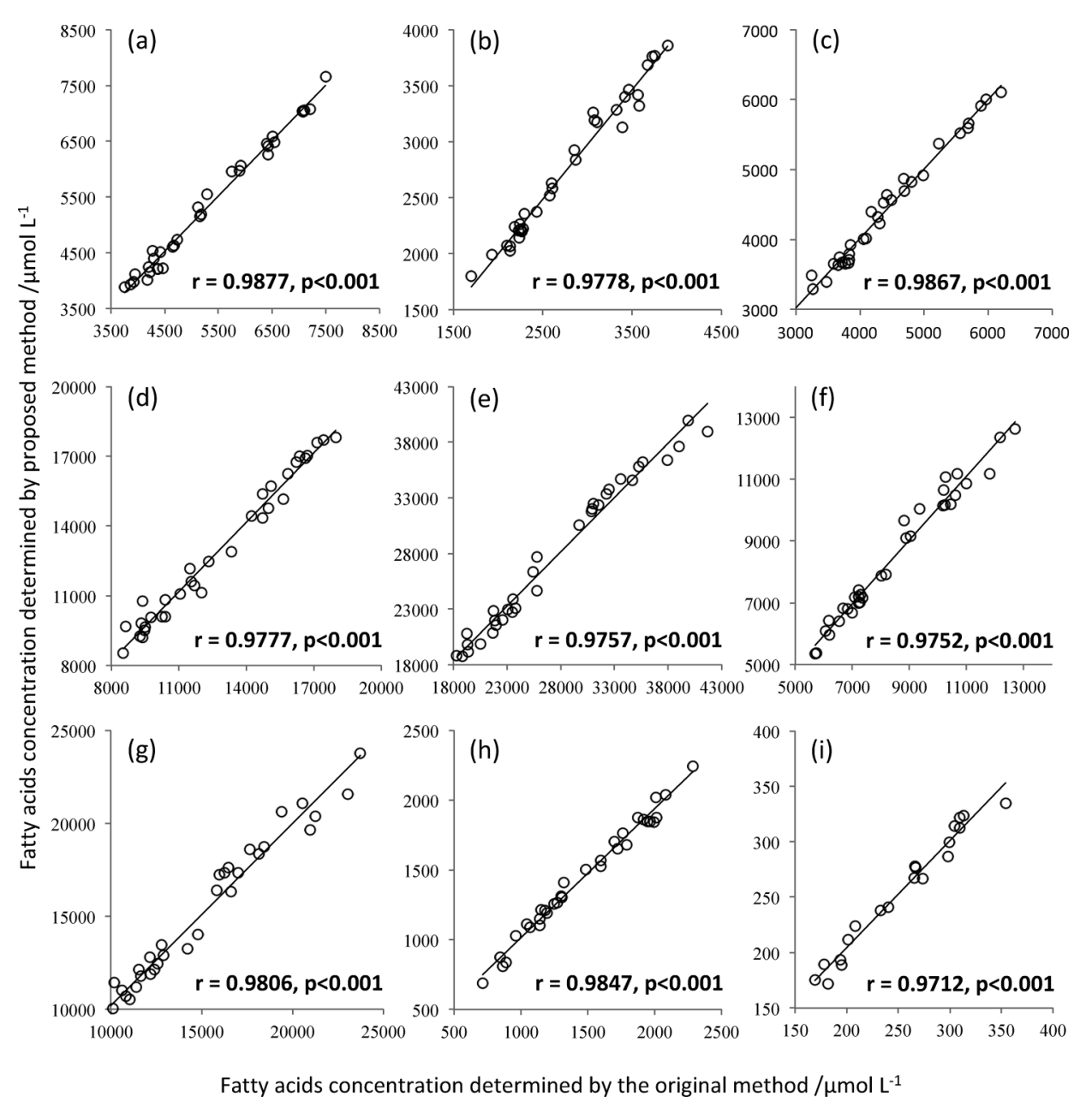

Fig. 4 Comparison of the fatty acid concentration between the proposed method and conventional heating method. (a) Hexanoic acid (FA6:0); (b) octanoic acid (FA8:0); (c) capric acid (FA10:0); (d) myristic acid (FA14:0); (e) palmitic acid (FA16:0); (f) stearic acid (FA18:0); (g) oleic acid (FA18:1); (h) linoleic acid (FA18:2); (i) arachidonic acid (FA20:4).

interfering substances that nearly co-elute with FA in HPLC. ${ }^{21}$ However, this step has been omitted in the current method, since we did not observe such interferences in the chromatogram following MAD. Thus, MAD not only has advantages of rapidity, but also minimizes the formation of byproducts.

Second, the MAD for the measurement of FA has a better analytical performance than the conventional method. MAD led to relatively increase yields for all investigated FA with a consequent improvement of the LOD. The recovery and reproducibility study demonstrated that the recovery of all fatty acids at 3 different concentrations ranged within $90-110 \%$, except for FA18:3 and CV of imprecision were within 10\% for FA (Table 1). Therefore, this method is suitable for the determination of FA concentrations in biological samples. We successfully applied this method for the measurement of the major FA present in milk. There was a significant agreement between the result obtained from MAD and conventional method with the correlation coefficient above 0.97 (Fig. 4). Based on these data, we concluded that the present method has satisfactory performance to measure the FA.

Another advantage of this method is its cost efficiency. The GC is time-consuming and requires considerable technical expertise. ${ }^{43}$ The proposed HPLC is technically simple and does not require expensive instrumentation. Furthermore, we used methanol as a solvent system for the HPLC separation of FA. Previous studies used acetonitrile as the solvent for HPLC separation, which is relatively expensive than methanol. ${ }^{14,16} \mathrm{We}$ found no difference in peak isolation and resolution between methanol and acetonitrile. As MAD decreases the reaction time avoiding prolong use of classical heating, it is considered as one of the approaches to greener derivatization in analytical laboratories. ${ }^{44}$ Therefore, our method of MAD for the quantification of FA is an environmental-friendly derivatization process.

Lastly, this method uses triglycerides as IS that can effectively evaluate the completion of saponification. As the majority of FA in the milk samples exist in esterified form, using triglycerides (TG11:0/11:0/11:0, TG19:0/19:0/19:0) as IS is superior to using free FA. We synthesized the triglycerides IS in our laboratory and to the best of our knowledge this is the first approach to use triglycerides as IS for the measurement of FA in the milk sample. Use of three IS to cover a wide range of carbon chain in FA also adds to the superiority of this method. Most previous methods for the determination of FA use a single

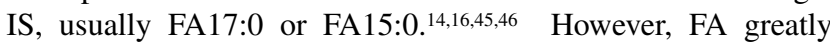
varies in the physical and chemical properties depending on the number of carbon in its chain. Therefore, the rate of the reaction during the derivatization of FA19:0, its solubility in the 
extracting solvents, and the molar absorptivity in spectrometry may significantly differ from FA with a shorter carbon chain. In other words, FA17:0, or FA19:0 alone is not a suitable IS for the quantification of SCFA and MCFA. To address this issue, we used FA7:0, FA11:0 and FA19:0 as IS for the determination of SCFA, MCFA, and LCFA, respectively. Hence, our assay provides reliable measurements of SCFA and MCFA compared to the assays that use single IS. Moreover, the methyl ester derivatization of FA for GC analysis may not be suitable for MCFA and SCFA because such esters are relatively volatile, therefore are lost at least partially during the pre-analytical specimen processing. ${ }^{13}$ This is not a problem in most biological samples that contain no or relatively trace amount of SCFA and MCFA. However, this becomes significant in the analysis of FA in milk, since it contains a considerable amount of SCFA and MCFA, comprising $8-22 \%$ of all FA. ${ }^{12}$ In the recent years, due to the unique metabolic properties of MCFA compared to LCFA, many researchers are focusing on the possible use of MCFA as a dietary intervention to benefit various clinical conditions including obesity, ${ }^{5,6}$ metabolic syndrome and insulin resistance, ${ }^{8,9}$ liver disease, ${ }^{10}$ and cardiac diseases. ${ }^{7}$ In the ordinary human diet of most countries, only milk and its products contribute to dietary MCFA. Therefore, the precise measurement of MCFA in milk is desirable to study the dietary importance of MCFA. The selection of carbon chain-specific FA as IS and MAD make our method to be ideal for the measurement of SCFA and MCFA.

One limitation of this proposed method is the requirement of a longer HPLC elution time to obtain an isolated high-resolution peak of individual FA without any influence of interfering substances. However, unlike previously reported methods to use separate analysis of SCFA, our approach can measure FA with all ranges of carbon $(\mathrm{C} 4-\mathrm{C} 20)$ in a single HPLC run. Further, we believe that the use of LC-MS/MS can significantly reduce the RT of eluting FA without the effect of interference, which is our future interest.

\section{Conclusion}

An improved method for the quantification of FA has been developed using the rapid MAD procedure that was successfully integrated with HPLC for fatty acids profiling of milk samples. The application of microwave irradiation significantly reduced the sample preparation duration. The performance of the proposed microwave method was compared with the classical heating method, which is proved to be superior with respect to the analytical time, recovery, and performance. The procedure described in the present study is useful in the fatty acids profiling of milk and particularly valuable in the analysis of SCFA and MCFA.

\section{Acknowledgements}

This study was financially supported by Research Grant for Rare and Intractable Disease from the Ministry of Health, Labor, and Welfare of Japan, partially by a Grant-in-Aid for Scientific Research from the Japan Society for the Promotion of Science, Japan, and partly by the Regional Innovation Strategy Support Program, Sapporo Health Innovation "Smart-H”, of the Ministry of Education, Culture, Sports, Science and Technology, Japan.

\section{Supporting Information}

This material is available free of charge on the Web at http:// www.jsac.or.jp/analsci/.

\section{References}

1. W. S. Harris, M. Miller, A. P. Tighe, M. H. Davidson, and E. J. Schaefer, Atherosclerosis, 2008, 197, 12.

2. P. C. Calder, Mol. Nutr. Food Res., 2012, 56, 1073.

3. Y. Momiyama, Atherosclerosis, 2013, 231, 281.

4. A. Kotani, M. Watanabe, K. Yamamoto, F. Kusu, and H. Hakamata, Anal. Sci., 2016, 32, 1011.

5. H. Tsuji, M. Kasai, H. Takeuchi, M. Nakamura, M. Okazaki, and K. Kondo, J. Nutr., 2001, 131, 2853.

6. M. P. St-Onge, R. Ross, W. D. Parsons, and P. J. Jones, Obes. Res., 2003, 11, 395.

7. F. Labarthe, R. Gelinas, and C. Des Rosiers, Cardiovas. Drugs Ther, 2008, 22, 97.

8. K. Nagao and T. Yanagita, Pharmacol. Res., 2010, 61, 208.

9. S. Terada, S. Yamamoto, S. Sekine, and T. Aoyama, Nutrition, 2012, 28, 92.

10. M. J. Ronis, J. N. Baumgardner, N. Sharma, J. Vantrease, M. Ferguson, Y. Tong, X. Wu, M. A. Cleves, and T. M. Badger, Exp. Biol. Med. (Maywood), 2013, 238, 151.

11. R. G. Jensen, A. M. Ferris, and C. J. Lammi-Keefe, Annu. Rev. Nutr., 1992, 12, 417.

12. R. G. Jensen, J. Dairy Sci., 2002, 85, 295.

13. E. S. Lima and D. S. P. Abdalla, Anal. Chim. Acta, 2002, 465,81 .

14. H. Miwa, Anal. Chim. Acta, 2002, 465, 237.

15. I. Brondz, Anal. Chim. Acta, 2002, 465, 1.

16. H. Miwa, C. Hiyama, and M. Yamamoto, J. Chromatogr., $\mathbf{1 9 8 5}, 321,165$.

17. H. G. Dean, J. C. Bonser, and J. P. Gent, Clin. Chem., 1989, 35, 1945.

18. K. Funazo, M. Tanaka, Y. Yasaka, H. Takigawa, and T. Shono, J. Chromatogr. A, 1989, 481, 211.

19. Y. Yasaka, M. Tanaka, T. Shono, T. Tetsumi, and J. Katakawa, J. Chromatogr., 1990, 508, 133.

20. A. Mehta, A. M. Oeser, and M. G. Carlson, J. Chromatogr. $B$, 1998, 719, 9 .

21. R. Shrestha, S. P. Hui, H. Imai, S. Hashimoto, N. Uemura, S. Takeda, H. Fuda, A. Suzuki, S. Yamaguchi, K. Hirano, and H. Chiba, Ann. Clin. Biochem., 2015, 52, 588.

22. R. Shrestha, K. Hirano, A. Suzuki, S. Yamaguchi, Y. Miura, Y. Chen, M. Mizuta, H. Chiba, and S. P. Hui, Anal. Sci., 2017, 33, 1297.

23. A. Lew, P. O. Krutzik, M. E. Hart, and A. R. Chamberlin, J. Comb. Chem., 2002, 4, 95.

24. A. de la Hoz, A. Díaz-Ortiz, and A. Moreno, Chem. Soc. Rev., 2005, 34, 164.

25. B. A. Roberts and C. R. Strauss, Acc. Chem. Res., 2005, 38, 653.

26. V. Polshettiwar and R. S. Varma, Acc. Chem. Res., 2008, 41,629 .

27. A. K. Rathi, M. B. Gawande, R. Zboril, and R. S. Varma, Coord. Chem. Rev., 2015, 291, 68.

28. K. Morita, A. Kobayashi, H. Nagatani, and H. Imura, Anal. Sci., 2015, 31, 481.

29. B. L. Hayes, Aldrichim. Acta, 2004, 37, 66.

30. A. C. Silvério, S. C. Machado, V. B. Boralli, and I. Martins, J. Sep. Sci., 2015, 38, 2664. 
31. S. A. Ahmed and E. M. Soliman, Anal. Sci., 2015, 31, 1047.

32. B. Mandal, P.K. Sinha, R. Sen, and A. K. Mandal, Anal. Sci., 2016, 32, 571.

33. S. L. Söderholm, M. Damm, and C. O. Kappe, Mol. Divers., 2010, 14, 869 .

34. G. Casals, J. Marcos, O. J. Pozo, J. Alcaraz, M. J. Martínez de Osaba, and W. Jiménez, J. Chromatogr. B, 2014, 960, 8.

35. L. Cao, T. Deng, S. Liang, X. Tan, and J. Meng, Anal. Sci., 2014, 30, 759.

36. L. Amendola, F. Garribba, and F. Botrè, Anal. Chim. Acta, 2003, 489, 233

37. T. Y. Chu, C. H. Chang, Y. C. Liao, and Y. C. Chen, Talanta, 2001, 54, 1163.

38. M. Athanasios, L. Georgios, and K. Michael, Food Chem., 2007, 102, 606.

39. Z. Zhang, G. Xiong, G. Lie, and X. He, Anal. Sci., 2014, $16,221$.
40. J. A. Bowden and D. A. Ford, J. Chromatogr. B, 2011, 879, 1375.

41. L. Amendola, C. Colamonici, M. Mazzarino, and F. Botrè, Anal. Chim. Acta, 2003, 475, 125.

42. S. Strassnig, M. Gfrerer, and E. P. Lankmayr, J. Chromatogr. $B$, 2004, 813, 151 .

43. N. Núñez-Sánchez, A. L. Martínez-Marín, O. Polvillo, V. M. Fernández-Cabanás, J. Carrizosa, B. Urrutia, and J. M. Serradilla, Food Chem., 2016, 190, 244.

44. I. Lavilla, V. Romero, I. Costas, and C. Bendicho, Trends Anal. Chem., 2014, 61, 1.

45. I-M. Chung, J-K. Kim, I. Park, J-Y. Oh, and S-H. Kim, Food Chem., 2016, 196, 138.

46. J. Añorve-Morga, A. Castañeda-Ovando, A. Cepeda-Saez, A. D. Archibold, J. Jaimez-Ordaz, E. Contreras-López, L. G. González-Olivares, and J. L. Rodríguez-Rodríguez, Food Chem., 2015, 172, 456. 